\title{
Guy Lachapelle
}

Professeur titulaire, Département de Science politique, Université Concordia, Montréal

(2008)

\section{"Les Québécois et l’ALENA. L’enthousiasme refroidit.”}

\author{
Un document produit en version numérique par Jean-Marie Tremblay, bénévole, \\ professeur de sociologie au Cégep de Chicoutimi \\ Courriel: jean-marie tremblay@uqac.ca \\ Site web pédagogique : http://www.uqac.ca/jmt-sociologue/ \\ Dans le cadre de: "Les classiques des sciences sociales" \\ Une bibliothèque numérique fondée et dirigée par Jean-Marie Tremblay, \\ professeur de sociologie au Cégep de Chicoutimi \\ Site web: http://classiques.uqac.ca/ \\ Une collection développée en collaboration avec la Bibliothèque \\ Paul-Émile-Boulet de l'Université du Québec à Chicoutimi \\ Site web: http://bibliotheque.uqac.ca/
}




\section{Politique d'utilisation de la bibliothèque des Classiques}

Toute reproduction et rediffusion de nos fichiers est interdite, même avec la mention de leur provenance, sans l'autorisation formelle, écrite, du fondateur des Classiques des sciences sociales, Jean-Marie Tremblay, sociologue.

Les fichiers des Classiques des sciences sociales ne peuvent sans autorisation formelle:

- être hébergés (en fichier ou page web, en totalité ou en partie) sur un serveur autre que celui des Classiques.

- servir de base de travail à un autre fichier modifié ensuite par tout autre moyen (couleur, police, mise en page, extraits, support, etc...),

Les fichiers (.html, .doc, .pdf., .rtf, .jpg, .gif) disponibles sur le site Les Classiques des sciences sociales sont la propriété des Classiques des sciences sociales, un organisme à but non lucratif composé exclusivement de bénévoles.

Ils sont disponibles pour une utilisation intellectuelle et personnelle et, en aucun cas, commerciale. Toute utilisation à des fins commerciales des fichiers sur ce site est strictement interdite et toute rediffusion est également strictement interdite.

L'accès à notre travail est libre et gratuit à tous les utilisateurs. C'est notre mission.

Jean-Marie Tremblay, sociologue

Fondateur et Président-directeur général, LES CLASSIQUES DES SCIENCES SOCIALES. 
Cette édition électronique a été réalisée par Jean-Marie Tremblay, bénévole, professeur de sociologie au Cégep de Chicoutimi à partir de :

Guy Lachapelle [Professeur titulaire, Département de Science politique, Université Concordia, Montréal.]

“Les Québécois et l’ALENA. L’enthousiasme refroidit”.

Un article publié dans Le Devoir, Montréal, édition du mercredi 23 avril 2008, page A9—idées.

[Autorisation formelle accordée par l'auteur le 23 avril 2008 de diffuser ce texte dans Les Classiques des sciences sociales.]

Courriel : guy.lachapelle@concordia.ca

Polices de caractères utilisée :

Pour le texte: Times New Roman, 14 points.

Pour les citations : Times New Roman, 12 points.

Pour les notes de bas de page : Times New Roman, 12 points.

Édition électronique réalisée avec le traitement de textes Microsoft Word 2004 pour Macintosh.

Mise en page sur papier format : LETTRE (US letter), 8.5'’ x 11'’)

Édition numérique réalisée le 23 avril 2008 à Chicoutimi, Ville de Saguenay, province de Québec, Canada.

\section{Fait avec}

Macintosh 
Guy Lachapelle

Professeur titulaire, Département de Science politique, Université Concordia, Montréal

“Les Québécois et l’ALENA. L’enthousiasme refroidit”.

Un article publié dans Le Devoir, Montréal, édition du mercredi 23 avril 2008, page A9—idées.

\section{Mots clés : Sommet des leaders d'Amérique du Nord, ALENA, Québec (province)}

La quatrième rencontre entre des chefs d'État de l'Amérique du Nord, qui a lieu à La Nouvelle-Orléans, offre une occasion de réfléchir non seulement aux progrès accomplis depuis l'entrée en vigueur de l'ALE et subséquemment de l'ALENA, il y a une vingtaine d'années, mais aussi aux défis des prochaines années.

Pendant que les candidats démocrates Hillary Clinton et Barack Obama décrient sans relâche les affres du libre-échange dans un climat électoraliste, on peut s'interroger sur l'évolution de l'attitude des Québécois envers le libre-échange en l'espace d'une dizaine d'années.

\section{De 1997 à 2007}

Pour mesurer ce changement, nous avons décidé de mener une nouvelle enquête à la fin de 2007 et de comparer les résultats avec ceux de notre enquête de 1997 (Groupe de recherche sur l'américanité). L'opération devait permettre de répondre à la question suivante: dix ans plus tard, que pensent les Québécois de l'ALENA? 
En 1997, l'euphorie entourant l'accord de libre-échange nordaméricain battait son plein -- on parlait même d'une ZLEA des Amériques -- alors qu'en 2007, la poussière de Ground Zero continuait de peser lourdement sur notre environnement économique et politique immédiat.

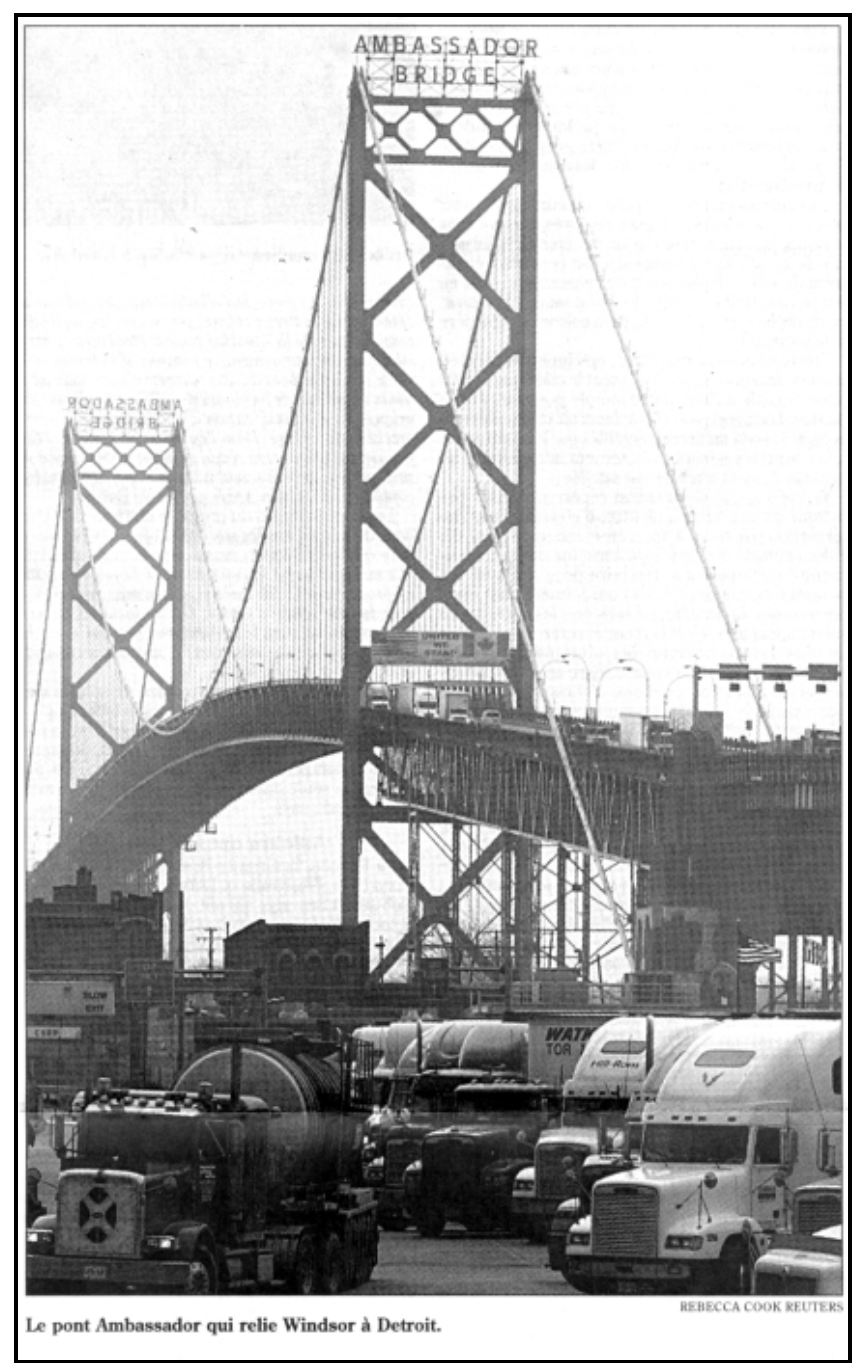

Photo : Le pont Ambassador qui relie Windsor à Detroit. Rebecca Cook, Reuters.

Les attentats du 11-Septembre ont profondément redéfini l'appartenance des Québécois au continent nord-américain. Si, en 2001, « nous étions tous des Américains ", la politique étrangère de l'administration 
américaine a profondément modifié les perceptions et opinions à l'endroit des États-Unis, non seulement au Québec mais partout dans le monde. Dans le contexte québécois, les décisions ou indécisions de l'administration Bush en ce qui concerne certaines politiques continentales (bois d'oeuvre, vache folle, commerce bilatéral, sécurité aux frontières, immigration) ont obligé les dirigeants politiques et les citoyens à réévaluer leurs attitudes envers les États-Unis.

\section{Points de vue}

L'évaluation de l'impact de l'ALENA, tout particulièrement sur les relations québéco-américaines, n'est pas toujours facile à faire selon qu'on se place d'un point de vue économique, culturel ou idéologique. Sur le plan économique, il ne fait aucun doute qu'il y a plusieurs points positifs. Comme l'écrivait le journaliste Éric Desrosiers dans Le Devoir en juin 2007 : «Le traité a pourtant été, de manière générale, un succès. Le volume de commerce et les investissements entre les trois pays ont augmenté. La production de plusieurs grandes entreprises et de secteurs de l'économie a été intégrée sur une base continentale. Une transition démocratique a été favorisée au Mexique. Les relations commerciales entre les trois pays ont été harmonisées. »

En fait, et contrairement à ce qu'annonçaient les détracteurs du libre-échange à la fin des années 1980, surtout au Canada anglais, les grandes catastrophes envisagées -- par exemple, la privatisation du système de santé ou l'intégration culturelle -- ne se sont pas produites. $[\ldots]$

Les données du commerce témoignent d'ailleurs assez éloquemment du chemin parcouru au cours des 20 dernières années. Toutefois, les événements du 11-Septembre ont eu un impact considérable tant sur les exportations que sur les importations canadiennes et québécoises vers les États-Unis. Ainsi, entre 2001 et 2006, les exportations ca- 
nadiennes sont passées de 87,0 à 81,6\%, et les importations, de 63,6 à $54,9 \%$.

C'est dans ce contexte de réduction des échanges commerciaux que lors du Sommet de Waco, en mars 2005, les gouvernements des trois pays signataires de l'ALENA ont décidé de créer un nouvel accord, le Partenariat nord-américain sur la sécurité et la prospérité (PSP), afin d'améliorer la collaboration et la coordination des politiques et réglementations en matière de sécurité et de prospérité. [...]

\section{Moins enthousiastes}

Les résultats comparatifs de nos enquêtes de 1997 et de 2007 montrent bien que les Québécois, s'ils restent plutôt optimistes, sont malgré tout moins enthousiastes qu'ils ne l'étaient il y a dix ans par rapport au libre-échange nord-américain. Il faut mentionner que depuis quelque temps, surtout au début de l'année 2008 alors que les primaires ont cours aux États-Unis, l'ALENA a le dos large, les deux candidates démocrates affirmant qu'ils souhaitent une renégociation de l'accord du libre-échange.

Dans un tel contexte -- et surtout avec la mondialisation qui favorise l'ouverture de nouveaux marchés --, un protectionnisme accru risque de remettre en question les relations économiques du Québec avec son partenaire du Sud.

Notre enquête de 2007 soulève d'abord une question cruciale quant au véritable succès de l'ALENA et à la capacité des élites québécoises à faire valoir les mérites de ce traité commercial. Ainsi, pour $63 \%$ des Québécois, ce sont les États-Unis qui ont le plus bénéficié de l'ALENA alors que 20\% estiment que c'est le Mexique et que seulement $8 \%$ mentionnent le Canada. Malgré tout, 55\% des Québécois (contre 62\% en 1997) estiment toujours que l'ALENA a eu un impact favorable sur le développement économique du Québec et que le marché américain 
demeure plus important que le marché canadien, et ce, à 54\% contre $30 \%$ (64\% contre $22 \%$ en 1997). [...]

\section{Intégration nord-américaine}

Malgré tout, les Québécois souhaitent, dans une proportion de $55 \%$, que le mouvement vers une plus grande intégration nordaméricaine continue au même rythme; 15\% aimeraient qu'il s'accélère alors que $28 \%$ souhaiteraient un ralentissement. Ces chiffres sont à peu près les mêmes qu'en 1997 (57\%).

Cependant, il y a aujourd'hui un peu plus de répondants qui souhaitent voir ce mouvement ralentir (28\% contre $19 \%$ en 1997 ) et moins qui veulent le voir s'accélérer (15\% contre 21\%). En 1997, 40\% des Québécois croyaient à la nécessité de créer des liens plus étroits avec les Etats-Unis ; en 2007, cette proportion a chuté à 30\%.

De même, si 36\% des répondants en 1997 préféraient que le Québec maintienne un fort degré d'indépendance par rapport à notre voisin du Sud, cet énoncé reçoit aujourd'hui l'appui de 42\% des répondants. [...]

\section{Une méfiance accrue}

Tout comme en 1997, les Québécois continue de manifester une très grande assurance identitaire, bien qu'on sente avec le passage du temps une méfiance accrue. En 2007, 55\% des Québécois estiment toujours que l'ALENA ne constitue pas une menace à la culture québécoise alors que 44\% affirment le contraire. En 1997, nous avions obtenu des résultats à $65 \%$ contre $31 \%$.

En fait, il faut se demander si ces résultats ne traduisent pas une certaine inquiétude identitaire liée autant aux effets de la mondialisation qu'aux menaces contre une certaine diversité culturelle. Notre en- 
quête indique que le nombre de personnes qui sont d'accord avec l'idée selon laquelle la mondialisation de l'économie représente une menace à la diversité culturelle est passé en dix ans de 29 à $46 \%$ alors que le nombre de personnes qui était en désaccord a diminué de 65 à $50 \%$.

Les débats entourant la protection des produits culturels ont sans doute laissé de nombreux Québécois plus songeurs quant à la place des produits culturels et à propos de l'importance d'exclure ces derniers de toutes négociations commerciales. [...]

Malgré tout, les Québécois ne voient pas dans l'ALENA une menace contre nos politiques publiques et en particulier contre nos programmes sociaux. Ainsi, 57\% d'entre eux (contre 35\%) estiment que l'ALENA ne constitue pas un danger pour le maintien de programmes sociaux. [...]

\section{Marché américain}

Notre enquête de 2007 démontre qu'une forte proportion de Québécois estiment toujours (à 54\% contre 30\%) que le marché américain demeure essentiel et plus important que le marché canadien pour l'avenir des exportations québécoises. Si l'optimisme était palpable en 1997 -- 64\% contre 22\% pour le marché américain --, on ne peut que noter ici encore un fléchissement de l'optimisme au cours de la dernière décennie. Un bon indicateur de ce ralentissement se perçoit dans le fait que 51\% des Québécois se disent en 2007 en désaccord avec l'idée d'avoir une monnaie commune en Amérique du Nord alors que 45\% y sont favorables.

Malgré tout, les Québécois souhaitent que les liens économiques du Québec avec les États-Unis s'intensifient dans le secteur touristique (69\%) et en matière de politiques environnementales (61\%). Ils souhaitent également (à 48\% contre 21\%) une intensification de nos ex- 
portations d'électricité, ce qui est nettement moins qu'en 1997 alors que $60 \%$ y étaient favorables, contre $9 \%$.

En matière d'éducation, si en 1997 les Québécois se disaient favorables à 55\% à des liens de coopération plus étroits en éducation, dix ans plus tard, ils sont plutôt partagés puisque $43 \%$ estiment que ces liens devraient rester les mêmes, 40\% qu'ils devraient s'intensifier et $14 \%$ qu'ils devraient diminuer. [...]

\section{Besoin d'enthousiasme}

Si l'ALENA a eu un impact certainement positif sur les relations commerciales entre le Québec et les États-Unis, il reste encore beaucoup de travail à faire pour redynamiser les relations commerciales et politiques. La comparaison entre les résultats de nos enquêtes de 1997 et de 2007 montre clairement que l'enthousiasme est moins palpable et qu'il y a nécessité pour les élites politiques des deux côtés de la frontière de réévaluer leurs actions.

Si le Partenariat pour la sécurité et la prospérité se voulait une réponse aux nombreux défis de l'après-11-Septembre, il reste beaucoup de travail à accomplir afin d'harmoniser les politiques continentales et d'accroître la qualité de vie des citoyens des deux pays. Les Québécois sont conscients de l'importance de leurs liens états-uniens et américains tout en affichant le plus grand réalisme quant aux nouveaux défis et aux occasions favorables au développement de nouveaux liens économiques.

\section{Fin du texte}

